\title{
Timber gridshells: Numerical simulation, design and construction of a full scale structure
}

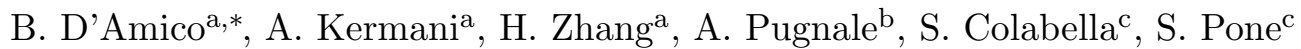 \\ ${ }^{a}$ School of Engineering and the Built Environment, Edinburgh Napier University, Edinburgh, UK \\ ${ }^{b}$ Faculty of Architecture, Building and Planning, University of Melbourne, Melbourne, Australia \\ ${ }^{c}$ Department of Architecture, University of Naples Federico II, Naples, Italy
}

\begin{abstract}
Timber gridshell structures, such as the Multihalle for the federal garden festival in Mannheim or the Downland Museum, have been the result of a creativegenerative process that indissolubly 'welded' the structural contribution to that of form exploration. The challenging design and construction issues have typically been addressed and resolved in several inventive but tedious steps and still till now, form-finding and erection of timber gridshells present difficulties that require radical solutions. In this regard, this paper aims to provide a series of novel steps to address some of the main design and construction issues that are associated with 'actively-bent' timber gridshell structures. First, the main characteristics of the construction process of timber gridshells are described and the basic theoretical concepts for its numerical simulation, through Dynamic Relaxation method, are introduced. Second, a practical method for sizing the laths' cross-section is presented. Third, a new erection technique for timber gridshells is proposed and applied to the construction of a full scale (prototype) structure, the Toledo gridshell 2.0. Fourth, a new bracing system for the same structure, which was built at the Faculty of Architecture, University of Naples Federico II in June-July 2014, is explained and discussed. The paper also highlights the need for further application to validate the techniques explained here, with particular attention being paid for the construction of large scale free-form structures.
\end{abstract}

\footnotetext{
${ }^{*}$ Corresponding author, telephone: +44 (0) 1314552249

Email addresses: bernardinodamico@gmail.com, b.d'amico@napier.ac.uk (B. D'Amico), a.kermani@napier.ac.uk (A. Kermani), j.zhang@napier.ac.uk (H. Zhang), alberto.pugnale@unimelb.edu.au (A. Pugnale), sofiacolabella@gmail.com (S. Colabella), sergio.pone@unina.it (S. Pone)
} 
Keywords: Gridshell, Active-bending, Timber, Dynamic Relaxation, Computational Form-finding.

\section{Issues of post-formed timber gridshells}

The term 'active-bending' refers to those structural systems in which a curved geometry is obtained through bending of elastic elements. It does not define a structural typology in itself. Rather, it groups different design approaches of constructing through bending. A detailed categorization is provided in [1] where a synoptic table relates forms and geometries to such approaches. In this paper, focus is only given to the particular construction system of gridshells - pre-assembled flat quadrilateral grids of straight and continuous elastic rods, which are subsequently post-formed, that is to say bent, into doubly-curved form-resistant shells (see, for instance, Trio gridshell in Figure 1). In fact, the doubly-curved - initially flat - gridshell, undergoes extentional (in-plane) deformations as a combination of the rods' bending and shear deformation of the initially square quadrilaterals. Such a 'pantograph kinematics' is prevented to occur at completion of the erection process by diagonal bracing systems, triangulating the quadrangular grid geometry. A review of relevant post-formed gridshell projects can be found in [2]. The described construction method generates structural forms by using standardised connection systems, such as single-bolts $[3,4]$ or clamping plates and devices $[5,6]$. However, such a repeatable and feasible solution has still to deal with the definition of the gridshell form, as well as with the design of an erection technique to bend the starting shape. This means that both theoretical and practical issues can only be addressed through a preliminary form-finding procedure and simulation of the erection, or forming, process.

In addition to this, the assessment of a 'feasible' size for the cross-section of bending members has to be performed. For instance, for a bent rod made of an elastic material with a given modulus of elasticity and strength, we have that the higher the curvature to be reached, the lower the allowed thickness of the rod will be. However, such a thickness might not be sufficient to provide the necessary structural performance. In the design of the Mannheim timber gridshell for the federal garden festival [3] this issue was brilliantly overcame with a double-layer system of overlapping timber laths. Compared to a single-layer system with equiv- 
alent cross-sectional area, this solution allowed for tighter curvatures to be reached. Thus, at completion of the bending process, the sliding between overlapping laths was restrained hence greatly increasing the bending stiffness of the built-up member. Regardless of the number of layers, the allowable cross-section, still needs to be assessed for each and every single lath, especially at early/conceptual design of the structural form.

(a)

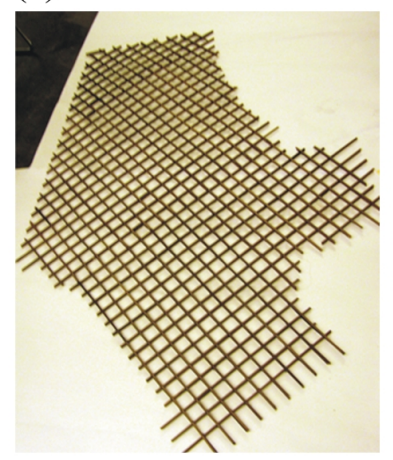

(b)

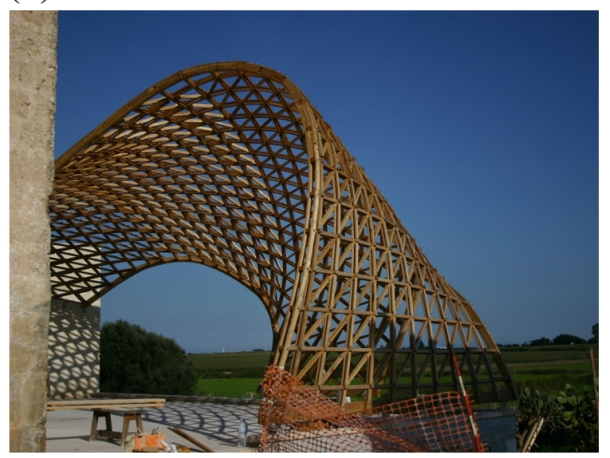

Figure 1: Trio gridshell in Lecce, Italy 2010 (CMMKM Architettura e Design): (a) Scale model of the flat grid; (b) Post-formed grid with diagonals bracing.

\section{A theoretical model}

The form of actively-bent structural systems cannot be chosen a priori. Rather, it will have to comply with the equilibrium of external (shaping) forces and internal reactions due to material and geometric stiffnesses. Accordingly, as for other kind of lightweight systems, as for instance tension structures [7] or reciprocal structures [8] some sort of preliminary form finding procedure is required.

Assuming, in first instance, the seeking of the post-formed gridshell geometry as a simulation of the construction process by means of Finite Element (FE) procedures, thus representing the geometry as a finite set $\mathbf{P}$ of nodes having nodal coordinates $\bar{p}_{i}$ in the Cartesian space:

$$
\mathbf{P}=\left\{\bar{p}_{1} \ldots \bar{p}_{i} \ldots \bar{p}_{m}\right\} \quad ; \quad \bar{p}_{i}=\left[\begin{array}{lll}
x & y & z
\end{array}\right]
$$

and a connectivity list $\mathbf{E}$ storing the node's indexes of the $j$ th beam-element's end 
nodes $(1,2)$ :

$$
\mathbf{E}=\left\{\mathbf{e}_{1} \ldots \mathbf{e}_{j} \ldots \mathbf{e}_{n}\right\} \quad ; \quad \mathbf{e}_{j}=\left\{i_{1}, i_{2}\right\}
$$

then, the form finding problem is reduced to solve the following system of equations:

50

$$
\mathbf{K} x=f
$$

where the vector of nodal displacements $\boldsymbol{x}$ from the unstressed initial position, corresponding, in our case, to the flat mat geometry, is obtained as function of the system stiffness matrix $\mathbf{K}$ and the vector of applied nodal forces $\boldsymbol{f}$ shaping the grid:

$$
\boldsymbol{x}=\mathbf{K}^{-1} \boldsymbol{f}
$$

In the 'general' case of small displacements theory, a linear relation is assumed between the displacement vector $\boldsymbol{x}$ and the load vector $\boldsymbol{f}$ and the problem is complied by computing the matrix K (Direct Stiffness Method [9]) according to the initial unstressed geometry. Such an approach is clearly unacceptable to simulate the large displacements involved in the forming process of elastic gridshells. Therefore, an iterative technique is required. The 'dominant' method in structural engineering for solving the system of non-linear equations (3) is known as the Transient Stiffness Method (TSM). As noted by Lewis [7] the method '...evolved from the conventional, small displacement theory', in that of keeping a linear relation between the vector of nodal forces and corresponding nodal displacements. However, unlike in the small displacements theory, the vector load is applied incrementally so that, the linearised displacements are 'corrected' and the stiffness matrix 'updated' at each increment (Full Newton-Raphson) in order to minimize the residual error (vector of out-of-balance forces) occurring as a consequence of the linearisation. Applications of the TSM for the form finding of post-formed timber gridshells (by means of Abaqus commercial software) are reported in [10, 11] (see Figure 1). In these, the simulation of the forming process allowed to find the gridshell geometry as well as assessing the resulting bending stress field, to be used as basis for the dimensioning of cross-section of the laths.

Clearly, a simulation of the forming process requires to know in advance the cutting pattern of the flat mat, corresponding to the initial unstressed geometry, as well as a vector of the external applied forces (or imposed displacements) needing to shape the mat according to the desired doubly curved shape we are looking 
for. For instance, according to Harris et al. [5] to design the Downland gridshell, physical scale modelling was used to determine the vector of boundary conditions

for the form finding model, while the flat mat was (a priori) established to have a rectangular contour perimeter (cutting pattern). Without doubt, a form finding procedure allowing to find the grid cutting pattern as well (according to a desired final shape) would be preferable to a 'mere' simulation of the construction process. An effort to define a 'comprehensive' approach to the form finding of post-formed gridshells can be found in $[12,13,14,15,16,17]$. In these, the main highlight is the use of a reference surface (acting basically as a form-work) on which 'forcing' the elastic grid to be bent. Then, in a second analysis step, the grid geometry exceeding the reference surface is removed from the analysis (a cutting pattern is so found) as well as the reference surface, thus, boundary constrains are added to the system and, at equilibrium convergence, the system settles down in its final static equilibrium. Clearly, in order to perform the described method, the initial mat geometry (node list $\mathbf{P}$ ) will have to lie on the reference surface, meaning that, unlike for a mere simulation of the forming process (where initial and unstressed geometries are coinciding) the initial geometry, at starting of the non-linear analysis will likely be far enough from static equilibrium to be intractable by TSM schemes (lack of numerical convergence). Accordingly, an explicit Finite Element procedure, such as the Dynamic Relaxation (DR) may be more suitable to be implemented in form finding frameworks expecting the use of a reference surface.

\subsection{Dynamic Relaxation Method: basic concepts}

The DR is an iterative time-stepping marching scheme, according to which, the original system of non-linear Eqs. (3) is transformed into a system of equations of motion by introducing lumped nodal masses and viscous damping forces [18] needed to allow the system reaching a rest configuration:

$$
\mathrm{M} a+\mathrm{C} v+\mathbf{K} x=f
$$

where $\mathbf{M}$ and $\mathbf{C}$ are (diagonal) mass and damping matrices respectively, while $\boldsymbol{a}$ is the list of nodal accelerations and $\boldsymbol{v}$ the list of nodal velocities. Expressing Eq. (5) at time $t$ in the form:

$$
\mathbf{M} \boldsymbol{a}^{t}+\mathbf{C} \boldsymbol{v}^{t}=\mathbf{R}^{t}
$$


with $\mathbf{R}$ representing the list of out-of-balance (residual) nodal forces as resultant of applied loads $\boldsymbol{f}$ and member's internal reactions $\mathbf{K} \boldsymbol{x}$, the nodal displacements can be computed at each time increment by integration of the acceleration and velocity terms. Since the stiffness matrix $\mathbf{K}$ in Eq. (6) disappeared, the system can be solved at a node-by-node level (this is a characteristic feature of the DR method, making it particularly appealing for parallel computing schemes [19]). Therefore, a general time marching scheme can be written for the generic $i$ th node. Adopting for instance the Euler forward integration scheme [20]: according to Newton's second law of motion, the acceleration term $\bar{a}$ for the $i$ th node in the Cartesian space at time $t+\Delta t$ is:

$$
\bar{a}_{i}^{t+\Delta t}=\frac{\bar{R}_{i}^{t}}{m}
$$

with $m$ the (fictitious) lumped mass. Therefore, the velocity projected at time $t+\Delta t$ will be:

$$
\bar{v}_{i}^{t+\Delta t}=c \bar{v}_{i}^{t}+\bar{a}_{i}^{t+\Delta t} \Delta t
$$

with $c$ the viscous damping term. Finally, the nodal displacement (hence the position) is:

$$
\bar{x}_{i}^{t+\Delta t}=\bar{v}_{i}^{t+\Delta t} \Delta t \quad ; \quad \bar{p}_{i}^{t+\Delta t}=\bar{p}_{i}^{t}+\bar{x}_{i}^{t+\Delta t}
$$

Regardless of the adopted numerical integration method, an essential task in any DR scheme is the computing of the nodal out-of-balance force $\bar{R}_{i}$ at each time increment, which is obtained as vector summation of the external applied force $\bar{P}_{i}$ plus internal reactions (for effect of the deformed state at time $t$ ) of the $n^{\circ}$ elements surrounding the $i$ th node:

$$
\bar{R}_{i}^{t}=\bar{P}_{i}+\sum_{j=1}^{n^{\circ}} \bar{F}_{j}^{t}
$$

For cables or struts behaviour-like, $\bar{F}^{t}$ will be only a function of the element's axial stiffness and shortening/elongation state at time $t$. Such kind of DR formulations are used for instance for the analysis of pre-stressed cable networks [21] or the form finding of funicular shell/dome structures [22]. On the other hand, for the simulation of elastically flexible members (as in our case) free body shear forces, due to the element's bending stiffness, need to be taken into account in the calculation of $\bar{F}^{t}[23]$.

Mostly, the DR method is implemented by only considering three degrees of freedom (DoFs) corresponding to the translational components in the Cartesian 
space. Despite considering only translational DoFs, DR procedures that efficiently simulate mechanical properties usually associated to rotational DoFs (flexural and torsional stiffnesses) have been proposed $[23,24]$. In general, the theoretical assumptions, upon which these DR formulations are built, require limitations for the cross-sectional geometry or the 'natural' (unstressed) geometry of the members ${ }^{1}$. For this reason, a more comprehensive DR formulation (not restricted to the aforementioned limitations) with six DoFs is adopted, i.e. for the study-case described in Section 4.1.

\subsubsection{Dynamic Relaxation with Six DoFs per node}

Developments of beam-element with six DoFs and their resolution by DR scheme date back to [25, 26]. In more recent developments [17, 27] mainly based on the work of Williams, (which derivation can be found in [28]) a local reference frame $\left\{\bar{x}_{i}, \bar{y}_{i}, \bar{z}_{i}\right\}$ describing the element's cross-section orientation, is assumed at each beam's end nodes $\left(i_{1}, i_{2}\right)$. Indicating with $L_{0}, A, I_{x}, I_{y}, J, E$ and $G$ respectively: the element's unstressed length, cross-sectional area, second moments of area, torsional constant, Young's and shear moduli: the reaction forces and moments at each end of the beam-element, needed for the computing of the residuals, are computed at each time increment as follow [17]:

$$
\mathbf{f}=\left\{\mathbf{K}_{A}^{t}+\mathbf{K}_{B}\right\} \cdot \mathbf{d}
$$

where:

$$
\mathbf{f}=\left[\begin{array}{c}
N \\
M_{x, 1} \\
M_{x, 2} \\
M_{y, 1} \\
M_{y, 2} \\
M_{\varphi}
\end{array}\right] ; \quad \mathbf{d}=\left[\begin{array}{c}
1 \\
\theta_{x, 1} \\
\theta_{x, 2} \\
\theta_{y, 1} \\
\theta_{y, 2} \\
\varphi
\end{array}\right]
$$

\footnotetext{
${ }^{1}$ Let us consider the simple case of a straight beam submitted to a torque. There is no way to simulate the torsion by only taking into account the nodal displacements - They remain null.
} 


$$
\mathbf{K}_{A}^{t}=E A e\left[\begin{array}{cccccc}
1 / L_{0} & 0 & 0 & 0 & 0 & 0 \\
& 2 / 15 & -1 / 30 & 0 & 0 & 0 \\
& & 2 / 15 & 0 & 0 & 0 \\
& & & 2 / 15 & -1 / 30 & 0 \\
\text { Symmetric } & & & & 2 / 15 & 0 \\
& & & & & 0
\end{array}\right]
$$

$$
\mathbf{K}_{B}=\frac{1}{L_{0}}\left[\begin{array}{cccccc}
0 & 0 & 0 & 0 & 0 & 0 \\
& 4 E I_{x} & 2 E I_{x} & 0 & 0 & 0 \\
& 4 E I_{x} & 0 & 0 & 0 \\
& & 4 E I_{y} & 2 E I_{y} & 0 \\
& & & 4 E I_{y} & 0 \\
\text { Symmetric } & & & & G J
\end{array}\right]
$$

Noting that: the stiffness matrix $\mathbf{K}_{B}$ is only function of material properties and unstressed geometry of the beam, whilst $\mathbf{K}_{A}^{t}$ takes into account the contribution due to geometric stiffness, as a function of the element's shortening/elongation and bowing $(e)$ at each increment of time. The element's local scalar reactions provided by $\mathbf{f}$ in Eq. (11) are then transformed in global reaction forces $\bar{F}_{1}, \bar{F}_{2}$ and moments $\bar{M}_{1}, \bar{M}_{2}$ in the Cartesian space, thus obtaining the (global) free body shear reactions, missing at a local reference frame level. The global reactions forces and moments so obtained are summed up (see Eq. (10)) for each node according to the corresponding surrounding elements, therefore obtaining the residual force $\bar{R}_{i}^{t}$ and residual moment $\bar{H}_{i}^{t}$ at the $i$ th node. Accordingly, the orientation of static equilibrium for the local frame $\left\{\bar{x}_{i}, \bar{y}_{i}, \bar{z}_{i}\right\}$ is sought (in analogy with the position of static equilibrium of the $i$ th node: Eqs. $(7-9))$ by assuming a system of lumped moments of inertia $\jmath$ (rotational masses):

$$
\bar{a}_{i}^{t+\Delta t}=\frac{\bar{H}_{i}^{t}}{\jmath}
$$

with $\bar{a}_{i}$ indicating, this time, the pseudo-vector of rotational acceleration of the local frame around the global (Cartesian) directions.

For a more detailed description of the introduced DR formulation with Six DoFs adopted for this study, the reader is referred to [17, 29]. 

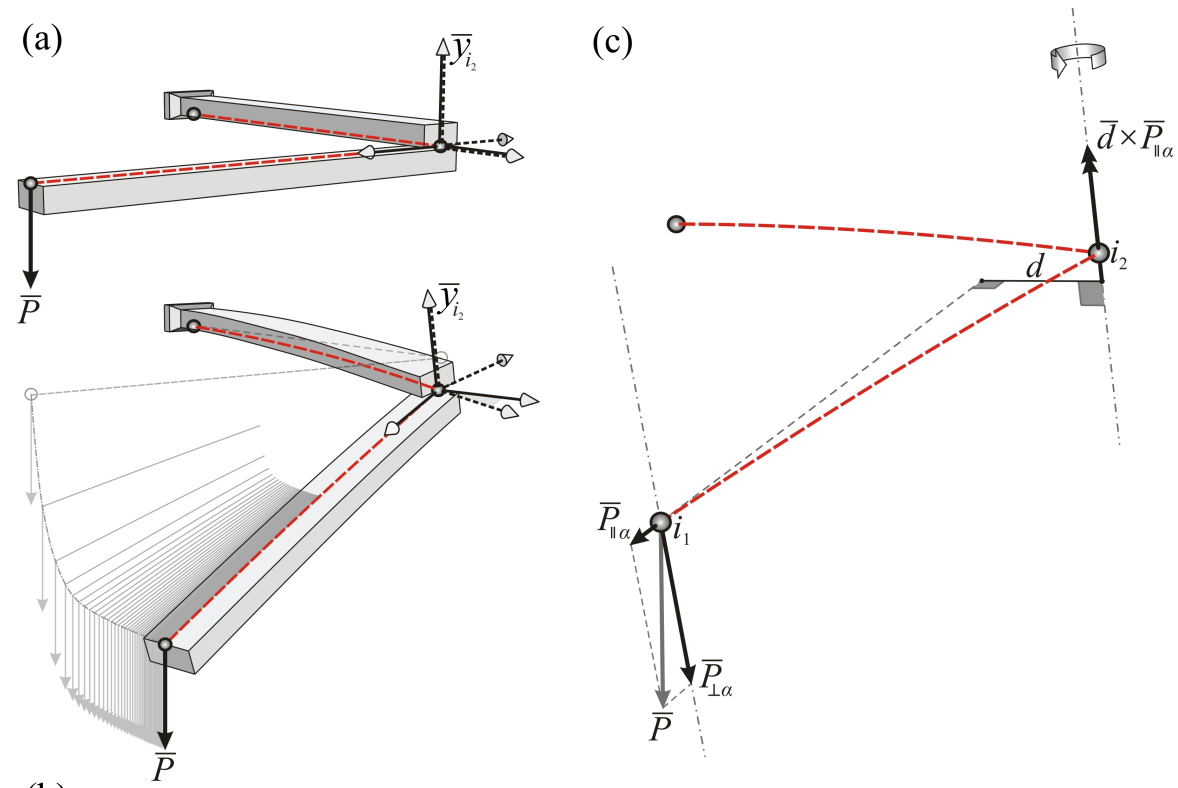

(b)

Figure 2: Single-node cylindrical joint: (a) Unloaded configuration; (b) Deformed configuration; (c) Decomposition of $\bar{P}$ in a parallel and an orthogonal component relative to the joint rotational axis.

\subsubsection{Cylindrical joints}

The presence of a local frame orientation at a node-level, ${ }^{2}$ consents to simulate

\footnotetext{
${ }^{2}$ In the Direct/Transient Stiffness Methods, a local reference frame is, in general, assumed at a element-level.
} 


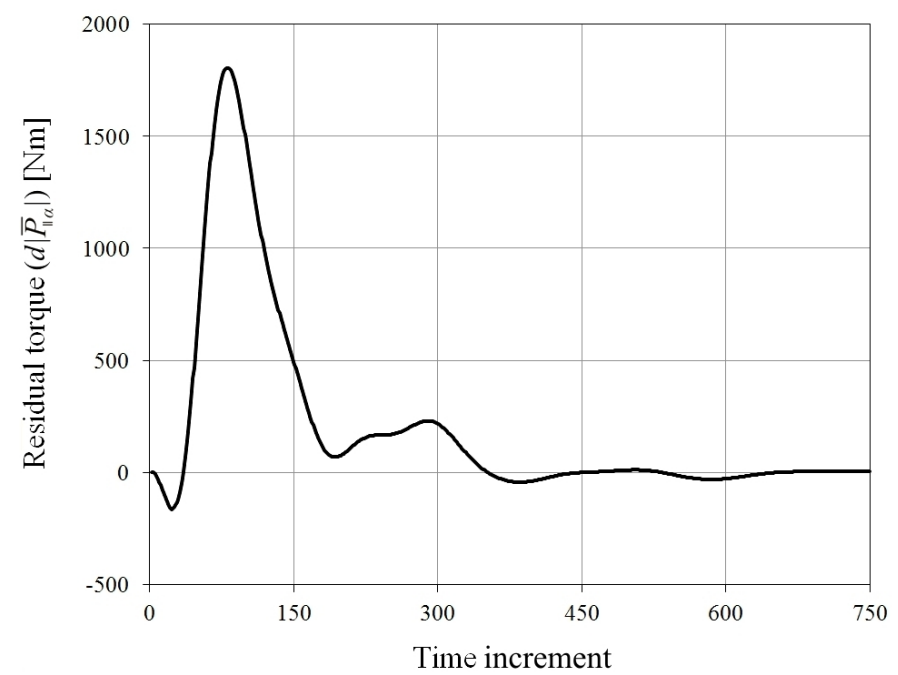

Figure 3: Time history of the out-of-balance torque $d\left|\bar{P}_{\| \alpha}\right|$ around the joint rotational axis

Indicating with $\bar{y}_{i_{2}}$ the joint's rotational axis at time $t$, and $\alpha$ the corresponding normal plane, the applied load $\bar{P}$ can be decomposed in two vectors:

$$
\bar{P}=\bar{P}_{\perp \alpha}^{t}+\bar{P}_{\| \alpha}^{t}
$$

Although magnitude and direction of load remain unchanged throughout the analysis, the joint's rotational axis does not. In fact, at initial configuration (Figure 2a) we have that $\bar{y}_{i_{2}}$ is aligned to $\bar{P}$, meaning that $\bar{P}_{\| \alpha}^{t=0}=\overline{0}$ thus no rotation occurs. However, as soon as the clamped rod starts bending and bowing (Figure 2b) the alignment between the vector load $\bar{P}$ and the joint rotational axis is lost, thus $\bar{P}_{\| \alpha}^{t}>\overline{0}$ and a rotation is triggered. In order for the system to reach a static equilibrium, sufficient condition is the nullification of the out-of-balance torque around the joint rotational axis:

$$
d\left|\bar{P}_{|| \alpha}\right| \approx 0
$$

where, $\bar{P}_{\| \alpha}$ can be computed at each time-step as:

$$
\bar{P}_{\| \alpha}^{t}=\bar{P}-\bar{P}_{\perp \alpha}^{t} \quad ; \quad \bar{P}_{\perp \alpha}^{t}=\left(\bar{P} \cdot \bar{y}_{i_{2}}^{t}\right) \bar{y}_{i_{2}}^{t}
$$

while the arm $d$ at time $t$ can be computed as the shortest distance between two skew lines [30]:

$$
d^{t}=\frac{\left(\bar{p}_{i_{2}}^{t}-\bar{p}_{i_{1}}^{t}\right) \cdot\left(\bar{P}_{\| \alpha}^{t} \times \bar{y}_{i_{2}}^{t}\right)}{\left|\bar{P}_{\| \alpha}^{t} \times \bar{y}_{i_{2}}^{t}\right|}
$$


Noting that Eq. (19) may provide negative values for $d^{t}$, depending on the orientation of the $\bar{P}_{\| \alpha}$ vector at time $t$.

According to the described set up, the time history of the out-of-balance torque $d\left|\bar{P}_{\| \alpha}\right|$ has been computed hence shown in Figure 3 as function of the time increment: As it can be seen, Eq. (17) is eventually fulfilled at the completion of the analysis.

\section{Computing the allowable cross-section}

As mentioned in the introduction: combined bending, arising for effect of the forming process, generates normal stress which imposes the cross-sectional size, of the single upper/lower laths, to be designed according to a certain domain's limit. In order to design the allowable cross-sectional height $h$ of a member subject to bending moment $M$, the following linear relation can be applied:

$$
h^{\text {allowable }}=\frac{2 I}{M} f_{m}
$$

with $f_{m}$ the material's bending strength. By expressing the bending moment in terms of curvature, Eq. (20) becomes:

$$
h^{\text {allowable }}=\frac{2}{E \kappa} f_{m}
$$

with the curvature $\kappa$ measured on the deformed geometry, obtained by assuming an initial guessing value for $h$. Accordingly, Eq. (21) states that, the curvature is only function of the assigned boundary displacements. While such an assumption remains valid for small displacements, in case of large displacements, the curvature is function of $h$ as well. In other words, when updating the value of $h$ as from Eq. (21), a change of $\kappa$ will occur as well, as a result of the updated bending stiffness of the member. Of course, Eq. (21) can be applied iteratively, by considering the updated curvature values at each iteration, up to a point for which the residual error becomes small enough to be neglected. A more detailed description for the suggested method, is given in the following Section.

\subsection{Theory}

In order to implement the described process, the ratios of combined bending 


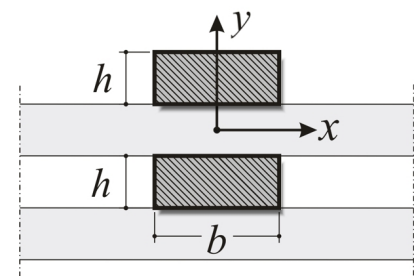

Figure 4: Local system orientation for the buit-up cross-section.

element set E. According to Eurocode 5 Part 1-1 [31] (EC5) the following (EC5 6.11 and 6.12 ) relations apply for combined bending:

$$
\frac{\sigma_{x}}{f_{m}}+k_{m} \frac{\sigma_{y}}{f_{m}} \leq 1 \quad ; \quad k_{m} \frac{\sigma_{x}}{f_{m}}+\frac{\sigma_{y}}{f_{m}} \leq 1
$$

therefore by assuming a limit stress ratio $=1$ and introducing a modification factor $\left(k_{m}=0.7\right.$ for rectangular cross sections [32]) to take into account the effects of variations in material properties and stress redistribution. ${ }^{3}$ The bending stress $\sigma_{x}$ and $\sigma_{y}$ are computed according to the local system orientation shown in Figure 4:

$$
\sigma_{x}=\frac{h\left|M_{x}\right|}{2 I_{x}} \quad ; \quad \sigma_{y}=\frac{b\left|M_{y}\right|}{2 I_{y}}
$$

Noting that, here we are only interested in assessing the thickness of the single lath to avoid breakages during the forming process, since, sliding between upper/lower laths is allowed at this stage of the construction. Accordingly, $I_{x}$ and $I_{y}$ in Eq. (23) relate to the cross-section of the single lath. Nevertheless, the required bending strength/stiffness of the built-up cross-section, in terms of load carrying capacity of the structure, can be provided in a second design stage, by sizing the thickness of the shear blocks (distance between upper/lower laths) which is directly proportional to the moment of area of the built-up cross-section.

Indicating with $\sigma_{j}^{6.11}$ and $\sigma_{j}^{6.12}$ the bending stress ratios of the $j$ th element as from Eqs. (22), we assume that, for a given post-formed geometry, as obtained from the form finding analysis, the maximum stress ratios $\sigma_{\max }^{6.11}$ and $\sigma_{\max }^{6.12}$ of the whole element set $\mathbf{E}$ :

$$
\sigma_{\max }=\max \left\{\sigma_{1} \ldots \sigma_{j} \ldots \sigma_{n}\right\}
$$

\footnotetext{
${ }^{3}$ In fact, timber shows a plastic behaviour under compression, while a brittle failure mode occurs under tension [33].
} 
can be represented by the values of two functions, whose argument $h$ was set to a certain value $h^{\mathrm{n}}$ :

$$
g\left(h^{\mathrm{n}}\right)=\sigma_{\text {max }}^{6.11} \quad ; \quad q\left(h^{\mathrm{n}}\right)=\sigma_{\max }^{6.12}
$$

Therefore, our aim is computing the value $h^{\text {allowable }}$ of the variable $h$ such that one of the two $g\left(h^{\mathrm{n}}\right)$ and $q\left(h^{\mathrm{n}}\right)$ is $\approx 1$ while the other one is less than the unity. Substituting Eqs. (23) into Eqs. (22) and rearranging, Eqs. (25) become:

$$
\begin{aligned}
& g(h)=\max \left(\frac{h\left|M_{x}\right|_{j}}{2 I_{x} f_{m}}+k_{m} \frac{b\left|M_{y}\right|_{j}}{2 I_{y} f_{m}}-1\right) \\
& q(h)=\max \left(k_{m} \frac{h\left|M_{x}\right|_{j}}{2 I_{x} f_{m}}+\frac{b\left|M_{y}\right|_{j}}{2 I_{y} f_{m}}-1\right)
\end{aligned}
$$

with $f_{m}$ a bending strength limit value. Accordingly, the problem is reduced to find the roots of the following system:

$$
h^{\text {allowable }}=\min \left\{\begin{array}{l}
h \rightarrow g(h)=0 \\
h \rightarrow q(h)=0
\end{array}\right.
$$

which can be numerically solved by Newton-Raphson method: expressing the second moments of area in Eqs. (26) in terms of $h$ and $b$, the following recurrence equation is obtained:

$$
\begin{gathered}
h^{\mathrm{n}+1}=\min \left\{\begin{array}{l}
h^{\mathrm{n}}-\frac{g\left(h^{\mathrm{n}}\right)}{\left(\frac{\partial g}{\partial h^{\mathrm{n}}}\right)} \\
h^{\mathrm{n}}-\frac{q\left(h^{\mathrm{n}}\right)}{\left(\frac{\partial q}{\partial h^{\mathrm{n}}}\right)}
\end{array}\right. \\
=\min \left\{\begin{array}{l}
\max \left[\frac{1}{\left|M_{x}^{\mathrm{n}}\right|_{j}}\left(\frac{\left(h^{\mathrm{n}}\right)^{3} f_{m} b}{6}-\frac{\left(h^{\mathrm{n}}\right)^{2} k_{m}\left|M_{y}^{\mathrm{n}}\right|_{j}}{b}\right)\right] \\
\max \left[\frac{1}{k_{m}\left|M_{x}^{\mathrm{n}}\right|_{j}}\left(\frac{\left(h^{\mathrm{n}}\right)^{3} f_{m} b}{6}-\frac{\left(h^{\mathrm{n}}\right)^{2}\left|M_{y}^{\mathrm{n}}\right|_{j}}{b}\right)\right]
\end{array}\right.
\end{gathered}
$$

Noting that the subscript $j$ refers to the generic element's index (see Eq. (2)) while the superscript ' $n$ ' refers to the nth analysis step (not to be confused with the size $n$ of the element set $\mathbf{E}$ ). A graphical representation of the iterative method is shown in Figure 5. 


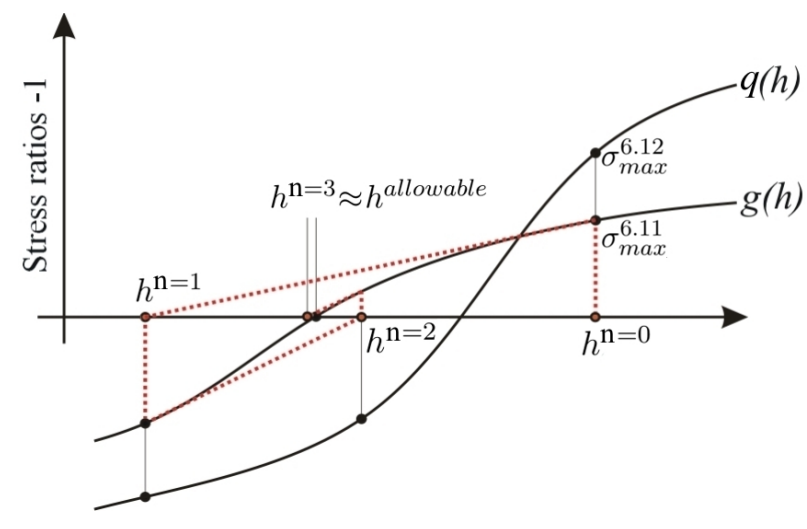

Figure 5: Newton-Raphson method for computing the allowable lath's thickness.

\section{Toledo Gridshell 2.0}

The theory described above was applied to a practical application in the forming process simulation and cross-sectional sizing of the Toledo 2.0, a post-formed timber gridshell built in 2014, the most recent of a series of 13 similar structures which have been designed and built by the research group gridshell.it. ${ }^{4}$ A description of the construction phases and their simulation by the six DoFs DR method is described in here, together with a brief report on the method's application for cross-sectional sizing detailed in section 3 .

\subsection{Construction process}

The structures by gridshell.it have been constructed in the following steps:

1. Assembling the flat grid of macro-moduli, which is placed and joined together on-site.

2. Deforming the flat grid to reach the final structural form by means of laths bending and relative rotation.

3. Restraining the boundary edges of the gridshell.

4. Placing the diagonal bracing system to ensure in-plane stiffness.

5. Tightening of the connection bolts to reach the final rigidity; (during the deforming phase, the bolts are kept untighten to allow the cylindrical hinge mechanism of the connections).

\footnotetext{
${ }^{4}$ gridshell.it is a research group based in Naples (Italy) led by Sergio Pone. Research partners are: Sofia Colabella, Bianca Parenti, Daniele Lancia.
} 

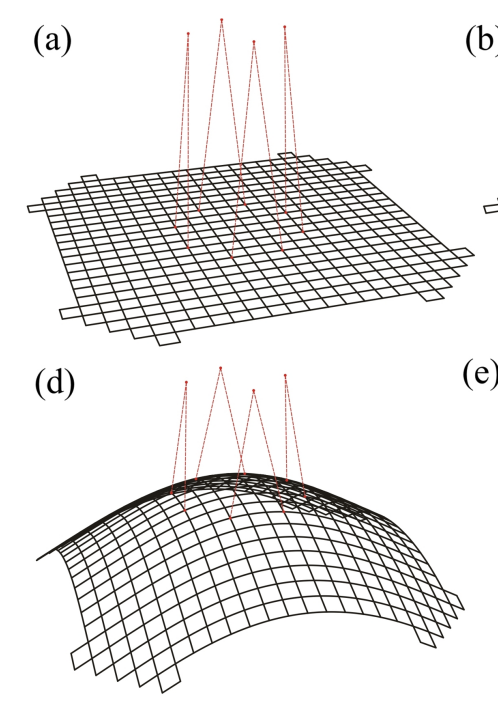

(b)

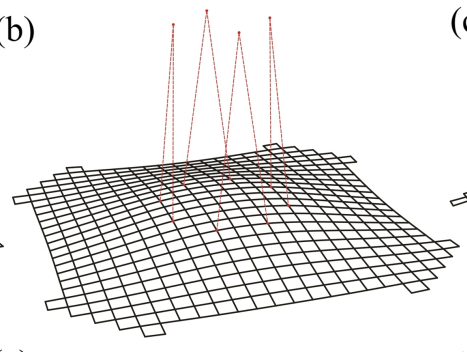

(e)

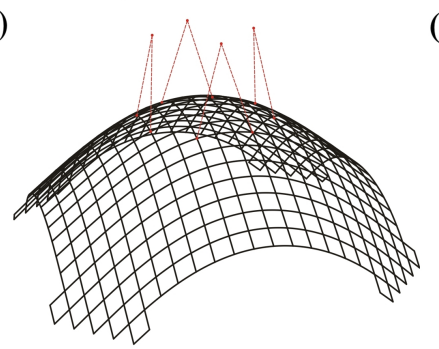

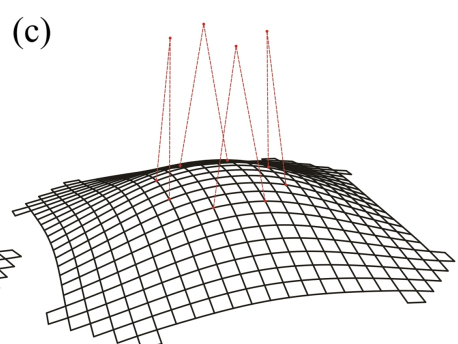

(f)

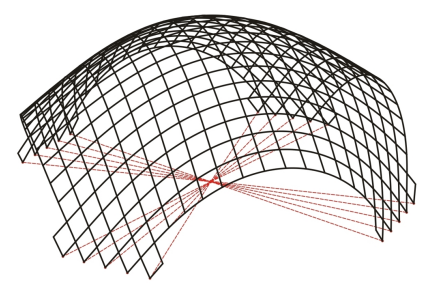

Figure 6: Toledo gridshell 2.0 in Naples, Italy 2014. Simulation of the forming process by DR: (a - e) The corner nodes are restrained on rollers while the central nodes are pulled up by pre-stressed cable-elements; (f) The cable-elements are 'disabled' from the analysis and additional horizontal trust is added to the corner nodes by means of pre-stressed cables.

Structural form of such gridshells can be obtained by using different tools and methods: from physical to numerical and hybrid ones. For the Toledo 2.0 it was used the Gridshell Form Finding Tool (GFFT) [34]: a computer application implemented within the plug-in for visual scripting Kangaroo [35] which is a commercial application based on the (explicit) resolution methods explained in Section. 2.1

The construction process of the Toledo 2.0 is based on the'up-ward' method. As for the Multihall in Mannheim [3], the flat mat was first assembled on ground and then forced to reach its final shape by means of ad-hoc machineries: As shown in Figures 6 and 7, eight central nodes of the gridshell were pulled up through a system made of pulleys placed on top of a scaffolding tower (placed at the centre of the flat grid) together with four cables for nautical use and four hand hoists. The grid was pulled up to a point for which the bracing system of the scaffolding tower, interfering with the grid, could not be avoided any further. At this stage, the interfering braces were temporary removed and reassembled in a new position underneath the grid. Once the final height of the gridshell was reached, two extra cross cables and hoists were added at the external nodes to provide horizontal trust, thus allowing to reach the desired shape (see Figures $6 \mathrm{f}$ and $7 \mathrm{~d}$ ). 
(a)

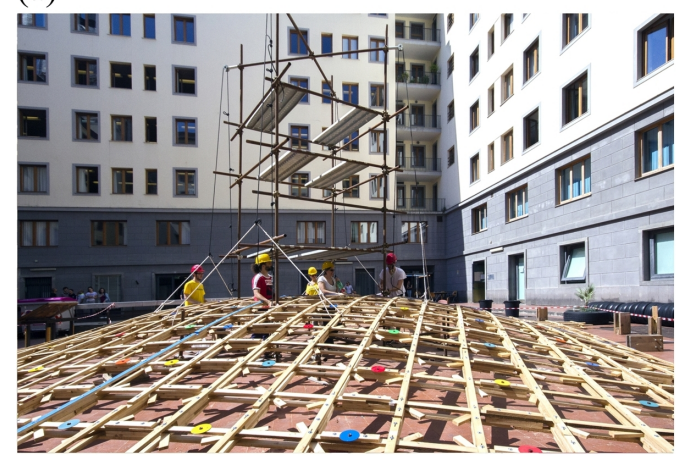

(c)

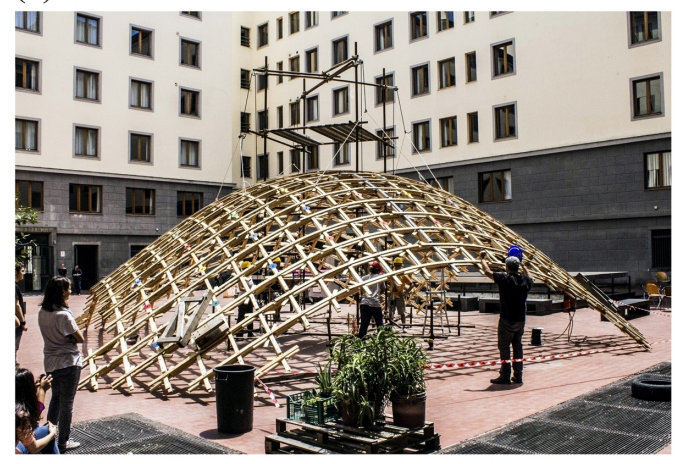

(b)

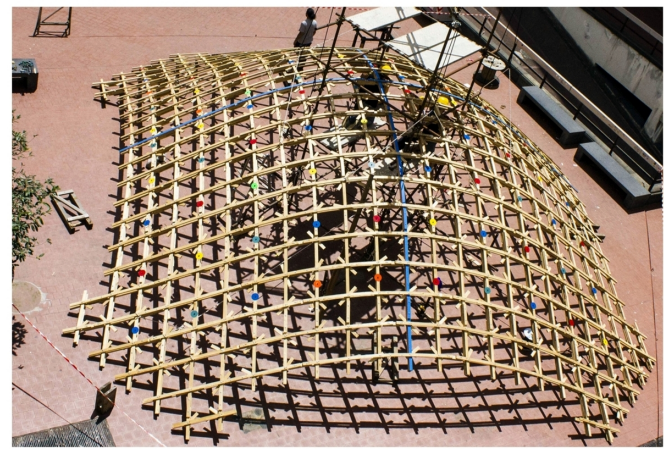

(d)

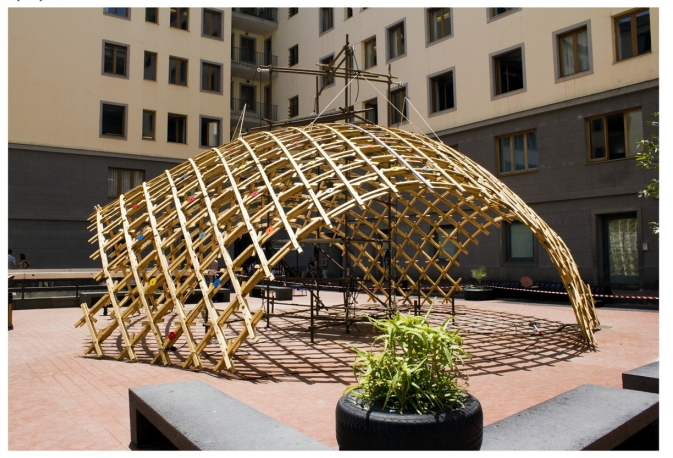

Figure 7: Toledo gridshell 2.0. Construction process: (a - c) The central nodes are pulled up by means of cables; (d) Additional horizontal trust is added to the corner nodes in order to reach the final shape.

The Toledo 2.0 was a successful test to 'tune' this experimental forming process, which we call: 'pull-up' method [2]. At completion of the bending process, the structural form was checked against the numerical analogue by electronic theodolite equipment, in a joint work with a research group affiliated to 'Suor Orsola Benincasa' University of Naples.

It is to be noted that the scaffolding tower assured safe working conditions during the whole erection process, by preventing sudden collapse of the grid - for example, due to unexpected breakage of a cable - and also allowing to conduct most of the operations from outside the grid footprint. The use of a localised support (tower) as a trust to pull-up the grid, opens up new possibilities for the construction of large span gridshells, i.e. by providing a 'discrete' system of fixedheight scaffolding towers, in contrast to more expensive 'continuous' systems of 
adjustable scaffolding. Further investigation needs to be carried on this particular aspect.

\subsection{Diagonal bracing system}

With the Toledo 2.0 there has also been the opportunity to experiment a new bracing system, consisting of the prefabrication of 'sticks' and 'diagonals', assembled every second quad of the grid. Short 'sticks' of timber were assembled at each nodal connection as a further layer (in addition to the 'usual' four) between the second and the third layer so that the first, second, fourth and fifth layers corresponded to the structural laths while the the middle (third) layer corresponded to the sticks (see Figure 8). The 'diagonals' were then lined up to the second and fourth layers, thus resembling a cross in between some of the quadrangles of the primary grid, (Figure 8b) eventually obtaining a chessboard-like pattern as shown in Figure 10.

Unlike the flat mat, in the formed gridshell each brace has a different length. In order to speed up the production/assembling, the numerical model was used to post-rationalise the bracing system by grouping the diagonals into only five (standardised) lengths and the sticks into three different lengths, thus assuring an overlapping distance (between sticks and diagonals) ranging from 70 to $120 \mathrm{~mm}$.

(a)

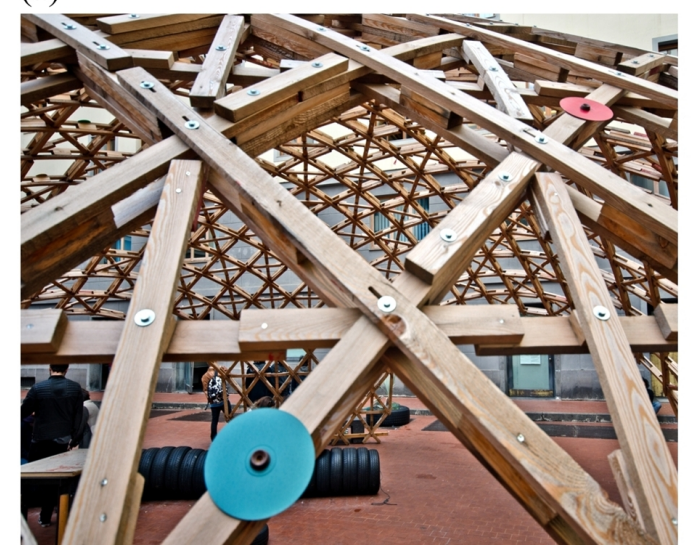

Figure 8: Toledo gridshell 2.0. Bracing system: (a) Construction detail; (b) Detail of the joint between sticks and diagonals. (b)

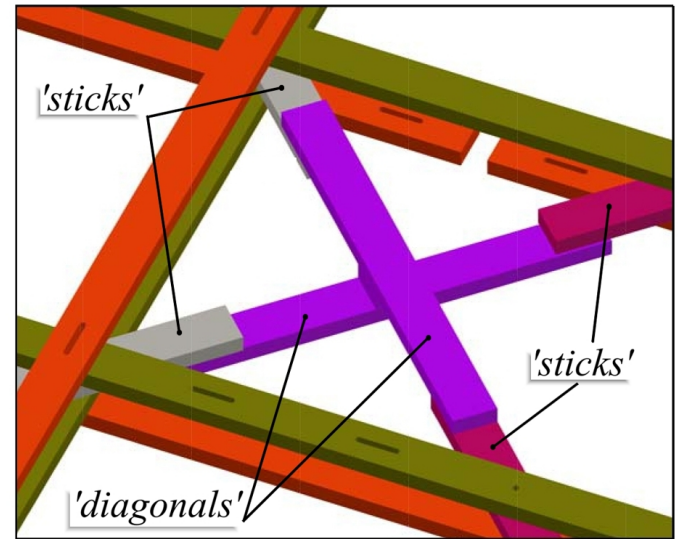




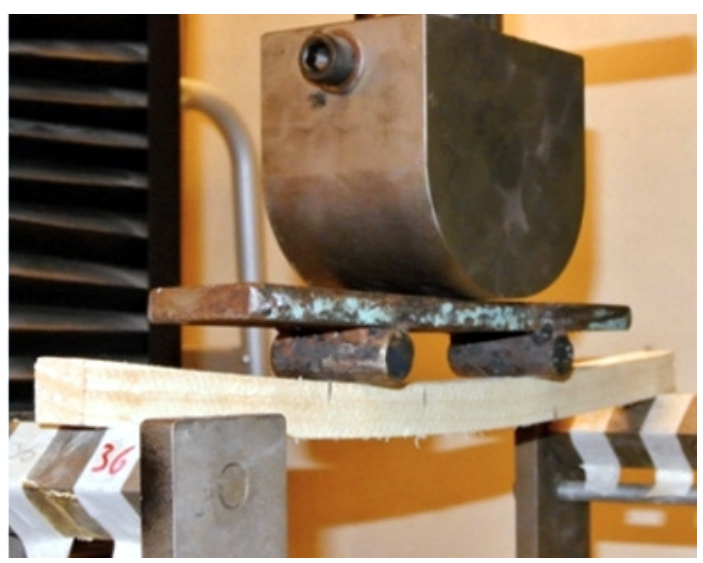

Figure 9: Four-point bending tests to assess the characteristic bending strength [11].

\subsection{Cross-sectional design}

The method described in Section 3 was applied in relation to the design of the Toledo 2.0 gridshell. Bending strength $\left(f_{m}\right)$ and elastic modulus $(E)$ were set in accordance to preliminary experimental investigations on the same white spruce timber, supplied for the realization of the Toledo gridshell 1.0 [11]. The value of $f_{m}$ to consider in Eq. (28) was set equal to the characteristic bending strength value $f_{m, k}=32 \mathrm{~N} / \mathrm{mm}^{2}$ obtained by preliminary bending tests on small, clear specimens, (see Figure 9) carried out in accordance to BS EN 408 guidelines [36]. A report of the test campaign is provided in [11]. The preliminary tests on small/clear specimens, were followed by visual inspection of the whole laths supply, in order make sure that 'defect-free' lath specimens were paced in correspondence of those regions of the flat mat where higher curvatures were expected to occur.

At completion of the forming process simulation, as described in Section 4.1, pinned restrains were added to the corner nodes and the iterative procedure for finding of the allowable thickness was performed. The moment reactions $M_{x}$ and $M_{y}$ needed for the calculation of the stress ratios (Eqs. (22)) were derived at the centroid of each element by applying the moment-curvature relations:

$$
M_{x}=\kappa_{x} E I_{x} \quad ; \quad M_{y}=\kappa_{y} E I_{y}
$$

with the curvature values $\kappa_{x}$ and $\kappa_{y}$ obtained from the element's shape function.

A report of the iterative process is given in Table 1: as it can be seen, despite an 'exaggerated' initial guessing value of $100 \mathrm{~mm}$, chosen for the lath's thickness, 
Table 1: Summary of the iterative method for computing of the allowable lath's thickness.

\begin{tabular}{cclr}
\hline Analysis Step & Lath's thickness $(h)[\mathrm{mm}]$ & \multicolumn{2}{c}{ Max. bending stress ratios } \\
\cline { 3 - 4 } & & $\sigma_{\max }^{6.11}$ & $\sigma_{\max }^{6.12}$ \\
\hline $1^{\text {st }}$ & 100.00 & 3.03 & 2.32 \\
$2^{\text {nd }}$ & 26.34 & 1.01 & 0.94 \\
$3^{\text {rd }}$ & 25.76 & 1.00 & 0.94 \\
$4^{\text {th }}$ & 25.68 & 1.00 & 0.94 \\
\hline
\end{tabular}

abased on Eqs. (26)

after only three iterations, the method provided an allowable value for $h=25.76$ $\mathrm{mm}$, corresponding to a maximum stress ratio of 1.0 with an order of accuracy up to the second decimal place. This suggests that the residual forces, arising for effect of the change in thickness (when passing from the analysis step $\mathrm{n}$ to the analysis step $\mathrm{n}+1$ ) are so small that a single step may be enough to computing a 'reasonable' accurate value for the allowable thickness $h$ : Inserting Eqs. (29) into Eqs. (23 - 22), Eq. (28) can be rewritten in terms of curvature values as:

$$
h^{\text {allowable }}=\min \left\{\begin{array}{l}
\max \left[\frac{1}{\left|\kappa_{x}\right|_{j}}\left(\frac{2 f_{m}}{E}-k_{m} b\left|\kappa_{y}\right|_{j}\right)\right] \\
\max \left[\frac{1}{k_{m}\left|\kappa_{x}\right|_{j}}\left(\frac{2 f_{m}}{E}-b\left|\kappa_{y}\right|_{j}\right)\right]
\end{array}\right.
$$

A final consideration is due in here with regard to the stresses induced by applied loads. Established that, external loads will act on the structure only 'after' the shear blocks have been inserted: the increase in bending stiffness, provided by these, needs to be taken into account when computing the load-induced stresses. In fact, the two overlapping laths will behave as a single, built-up, member. A model to simulate such a coupled behaviour is provided for instance in [29], according to which, the normal stresses at the external fibre of the built-up member (due to bending of the members for effect of applied loads) are obtained as summation of bending stress of the single lath plus another term due to the presence of shear blocks.

Furthermore, the stresses induced by applied loads will have to be combined with the pre-stress field induced by the forming process. In reality, the pre-stress field will reduce over time, as consequence of relaxation of timber under the effect of 
permanent displacements (that is to say: the forming process). Relaxation/creep of timber are phenomena leading to a reduction in strength and stiffness over time, as a result of accumulation of 'damages' at a cellular level. Such a complex material behaviour requires rheological (e. g. viscoelastic) models in order to be numerically simulated, however, a simplified but effective approach is 'allowed' by the EC5 [31] with regard to curved laminated beams. According to this, the prestress induced by the forming process can be 'neglected' when performing stress verification analyses of the structure under effect of applied loads (the gridshell geometry is assumed as being 'stress-free') but providing a reduced value for the design bending strength, which has to be scaled down by the following $k_{r}$ factor:

$$
0.76<k_{r} \leqslant 1
$$

Such a reduction factor will be a function of the lath's thickness and curvature (see Eq. (6.49) of EC5). Noting that: the EC5 allowance in neglecting the prestresses, only applies to the design task of carrying out stress verification analyses, whereas, if applied e.g. to buckling analyses, such an assumption may lead to an overestimation of the structure's global stability: In facts, it has been analytically and numerically demonstrated as the influence of pre-stress has a negative (lowering) effect on the critical buckling load of elastically bent arches [37, 38] and gridshells [39].

\section{Conclusions}

In this paper, the main theoretical and practical issues relating to a particular type of actively-bent system, (post-formed timber gridshells) have been described. In particular, the basic theoretical concepts to perform efficient form-finding and design/simulation of the construction (erection) process, have been illustrated, as well as a practical method for the sizing of the laths' cross-section.

The generalised six DoFs Dynamic Relaxation method adopted in here, allowed to efficiently simulate the 'full' mechanical behaviour of actively bent frameworks, made of elastic (timber) rods with rectangular cross-section, therefore overcoming the limitations usually associated with reduced DoFs formulations of rods, aimed to be solved by DR methods. As already pointed out in a previous paper [17]: thanks to its explicit 'nature', the adopted method allows for form-finding analyses to be 
performed in conditions of initial gross deformations, as well as simulations of the forming process in which, hyper-elastic elements can be accounted for simulating systems of pulling cables, without having to deal with numerical convergence issues (provided a stable mass/time-increment ratio). The method also allowed to simulate complex coupling systems, such as cylindrical joints, in a very simple way, by assuming only one node per connection, hence validating its accuracy by defining in here a benchmark numerical test (Section 2.1.2).

A second, major point addressed in here, regarded the development of a method for 'automatic' design of the laths' cross-sectional size. Starting from the theoretical assumption that, for a given deformed configuration, (outcome of imposed boundary displacements) a non-linear relation occurs between the thickness of the deformed (bent) laths and the curvature field along the laths' centerline (hence the corresponding bending stress field): an iterative procedure, based on NewtonRaphson method, has been developed to compute the allowable thickness of the laths' cross-sections, such that the highest bending stress/strength ratio turns out to be equal to the unity. An opportunity to test the described iterative method came out with the construction of a full scale prototype (the Toledo 2.0) in which, the non-linear nature of the thickness/curvature relationship was shown to be negligible. In facts, as reported in Table 1, the thickness values converged to an 'optimum' (allowable) value so rapidly to suggest the use of a linear expression (see Eq. (30)) for the determination of the allowable thickness.

The realization of the Toledo 2.0 (Figure 10) provided also the opportunity to experiment a novel bracing system, contemplating the use of two diagonal braces for each second quad of the grid, therefore having a chessboard-like pattern, with stiffened and non-stiffened quads alternating each other. If one brace per quad had been fitted on the total amount of quads, it would have presumably required the same amount of material. Nevertheless, by having two timber braces crossing each other, with a fastener (bolt) at their intersection (see Figure 8a) allowed to reduce the effective length of elastic buckling, thus obtaining a theoretical buckling load (of the braces under compression forces) four times higher than the one obtainable with a single brace of equal cross-section.

Further applications are needed to validate the techniques explained here, with a particular attention to design and construction of large scale, free-form structures. 

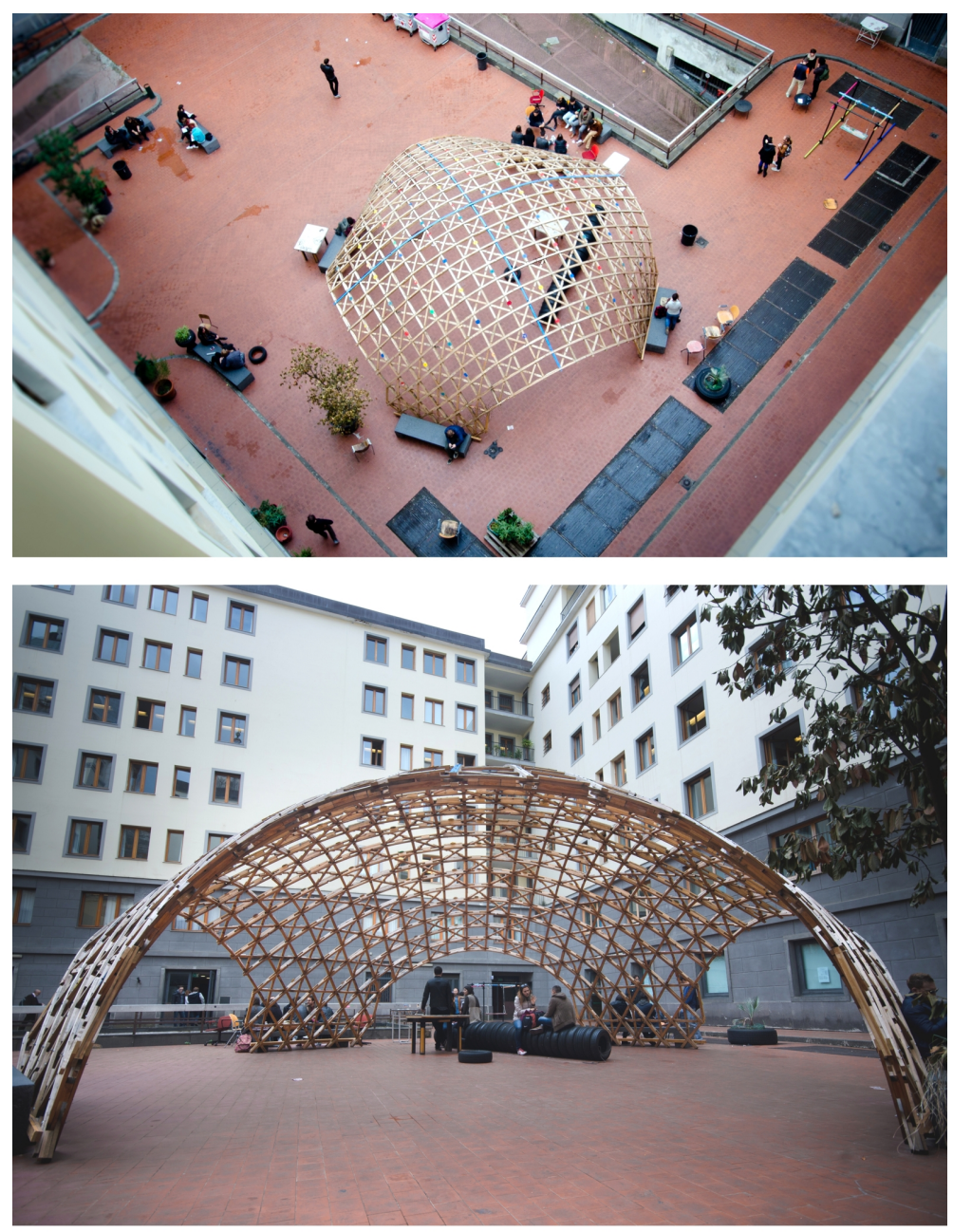

Figure 10: Toledo timber gridshell 2.0. Completed structure (Photos courtesy - Daniele Lancia).

\section{Acknowledgements}

The Centre for Timber Engineering (CTE) and the School of Engineering and Built Environment (SEBE) at Edinburgh Napier University, are gratefully acknowledged for providing financial support to the described research work.

\section{References}

[1] J. Lienhard, H. Alpermann, C. Gengnagel, J. Knippers, Active bending, a review on structures where bending is used as a self-formation process, In- 
ternational Journal of Space Structures 28 (3) (2013) 187-196. doi:http: //dx.doi.org/10.1260/0266-3511.28.3-4.187.

[2] G. Quinn, C. Gengnagel, A review of elastic grid shells, their erection methods and the potential use of pneumatic formwork, Mobile and Rapidly Assembled Structures IV 136 (2014) 129.

[3] E. Happold, W. Liddell, Timber lattice roof for the mannheim bundesgartenschau, The structural engineer 53 (3) (1975) 99-135.

[4] R. Harris, S. Haskins, J. Roynon, The savill garden gridshell: design and construction, The Structural Engineer 86 (17) (2008) 27-34.

[5] R. Harris, J. Romer, O. Kelly, S. Johnson, Design and construction of the downland gridshell, Building Research \& Information 31 (6) (2003) 427-454. doi:http://dx.doi.org/10.1080/0961321032000088007.

[6] C. Douthe, J.-F. Caron, O. Baverel, Gridshell structures in glass fibre reinforced polymers, Construction and building materials 24 (9) (2010) 1580-1589. doi:http://dx.doi.org/10.1016/j. conbuildmat.2010.02.037.

[7] W. J. Lewis, Tension structures: form and behaviour, Thomas Telford, 2003. doi:http://dx.doi.org/10.1680/tsfab.32361.

[8] C. Douthe, O. Baverel, Design of nexorades or reciprocal frame systems with the dynamic relaxation method, Computers \& Structures 87 (21) (2009) 12961307. doi:http://dx.doi.org/10.1016/j.compstruc.2009.06.011.

[9] K.-J. Bathe, Finite element procedures, Klaus-Jurgen Bathe, 2006.

[10] B. D'Amico, Design and building of a post-formed timber gridshell, Master's thesis, University of Naples Federico II, Italy (2010).

[11] S. Pone, B. D'Amico, S. Colabella, B. Parenti, D. Lancia, A. Fiore et al, Construction and form-finding of a post-formed timber grid-shell, in: Structures and architecture. Concepts, Applications and challenges, 2013, pp. 245-252. doi:http://dx.doi.org/10.1201/b15267-32. 
[12] L. Bouhaya, O. Baverel, J.-F. Caron, Mapping two-way continuous elastic grid on an imposed surface: Application to grid shells, in: Proc. 50th International IASS Symposium, Valencia, Editorial de la Universitat Politécnica de Valencia., 2010.

[13] J.-M. Li, J. Knippers, Form-finding of grid shells with continuous elastic rods, in: Proc. of the International Symposium of the IABSE-IASS Symposium, London, UK, Vol. 35, 2011.

[14] M. Kuijvenhoven, P. Hoogenboom, Particle-spring method for form finding grid shell structures consisting of flexible members, Journal of the International Association for Shell and Spatial Structures 53 (1) (2012) 31-38.

[15] O. Baverel, J.-F. Caron, F. Tayeb, L. D. Peloux, Gridshells in composite materials: Construction of a $300 \mathrm{mq}$ forum for the solidays' festival in paris, Structural Engineering International 22 (3) (2012) 408-414. doi:http://dx. doi .org/10.2749/101686612X13363869853572.

[16] B. DAmico, A. Kermani, H. Zhang, A form finding method for post formed timber grid shells, in: proceedings of the World Conference on Timber Engineering, Quebec City, Canada, 2014. doi:http://dx.doi.org/10.13140/2. 1.3949 .8888 .

[17] B. D'Amico, A. Kermani, H. Zhang, Form finding and structural analysis of actively bent timber grid shells, Engineering Structures. 81 (2014) 195-207. doi:http://dx.doi.org/10.1016/j.engstruct.2014.09.043.

[18] M. Papadrakakis, A method for the automatic evaluation of the dynamic relaxation parameters, Computer methods in applied mechanics and engineering 25 (1) (1981) 35-48. doi:http://dx.doi.org/10.1016/0045-7825(81) 90066-9.

[19] B. Topping, A. Khan, Parallel computation schemes for dynamic relaxation, Engineering computations 11 (6) (1994) 513-548. doi:http://dx.doi.org/ 10.1108/02644409410799407.

[20] K. E. Atkinson, An introduction to numerical analysis, John Wiley \& Sons, 2008. 
[21] M. Barnes, Form-finding and analysis of prestressed nets and membranes, Computers \& Structures 30 (3) (1988) 685-695. doi:http://dx.doi.org/ 10.1016/0045-7949(88) 90304-5.

[22] A. Kilian, J. Ochsendorf, Particle-spring systems for structural form finding, Journal of the International Assossation for Shell and Spatial Structures 148 (2005) 77.

[23] S. Adriaenssens, M. Barnes, Tensegrity spline beam and grid shell structures, Engineering structures 23 (1) (2001) 29-36. doi:http://dx.doi.org/10. 1016/S0141-0296(00)00019-5.

[24] M. R. Barnes, S. Adriaenssens, M. Krupka, A novel torsion/bending element for dynamic relaxation modeling, Computers \& Structures 119 (2013) 60-67. doi:http://dx.doi.org/10.1016/j.compstruc.2012.12.027.

[25] D. Wakefield, Dynamic relaxation analysis of pre-tensioned networks supported by compression arches., Ph.D. thesis, City University, London (1980).

[26] C. F. Ong, Computer aided design of tension structures., Ph.D. thesis, City University, London (1992).

[27] G. Senatore, D. Piker, Interactive real-time physics: An intuitive approach to form-finding and structural analysis for design and education, ComputerAided Design 61 (0) (2015) 32-41. doi : http://dx.doi.org/10.1016/j.cad. 2014.02 .007$.

[28] S. M. L. Adriaenssens, Stressed spline structures., Ph.D. thesis, University of Bath (2000).

[29] B. DAmico, A. Kermani, H. Zhang, P. Shepherd, C. J. K. Williams, Optimization of cross-section of actively bent grid shells with strength and geometric compatibility constraints, Computers \& Structures 154 (2015) 163 176. doi:http://dx.doi.org/10.1016/j.compstruc.2015.04.006.

[30] F. S. Hill, The Pleasures of Perp. Dot. Products. Ch. II.5 in Graphics Gems IV, Academic Press, 1994. 
[31] BS EN 1995-1-1:2004+A1:2008. Eurocode 5: Design of timber structures Part 1-1: General - Common rules and rules for buildings, British Standards Institution.

[32] J. Porteous, A. Kermani, Structural timber design to Eurocode 5, 2nd edition, John Wiley \& Sons, 2013.

[33] J. Bodig, B. A. Jayne, Mechanics of wood and wood composites, Van Nostrand Reinhold, 1981.

[34] S. Pone, S. Colabella, B. D'Amico, D. Lancia, A. Fiore, B. Parenti, Timber post-formed gridshell: Digital form-finding / drawing and building tool, in: Proc. of the IASS Symposium, Wroclaw, Poland, 2013. doi:http://dx.doi. org/10.13140/2.1.1339.1686.

[35] D. Piker, Kangaroo: Form finding with computational physics, Architectural Design (2013) 245-252.doi:http://dx.doi.org/10.1201/b15267-32.

[36] BS EN 408:2010+A1:2012. Timber structures. Structural timber and glued laminated timber. Determination of some physical and mechanical properties, British Standards Institution.

[37] J. Thompson, G. W. Hunt, On the buckling and imperfection-sensitivity of arches with and without prestress, International Journal of Solids and Structures 19 (5) (1983) 445-459. doi :dx.doi.org/10.1016/0020-7683(83) 90055-0.

[38] S. A. Chini, A. M. Wolde-Tinsae, Effect of prestressing on elastica arches, Journal of engineering mechanics 114 (10) (1988) 1791-1800. doi:http:// dx.doi.org/10.1061/(ASCE) 0733-9399(1988)114:10(1791).

[39] R. Mesnil, J. Ochsendorf, C. Douthe, Influence of the pre-stress on the buckling capacity of elastic grid shells, in: Proc. of the IASS Symposium, Wroclaw, Poland, 2013. 